\title{
Inteligencia competitiva, estrategia y competitividad de las empresas venezolanas
}

\author{
Pablo Lira"
}

\section{Resumen}

- Este artículo describe lo que el autor visualiza como el contexto de las nuevas realidades organizativas; el significado y la importancia de la Inteligencia Competitiva (IC); su relación con la gerencia estratégica; la descripción del modelo de la IC; algunos lineamientos para la instrumentación de una unidad de IC; la importancia del aspecto ético del proceso y las conclusiones. Este enfoque se fundamenta en las nuevas realidades tecnológicas, económicas y gerenciales de las empresas venezolanas y latinoamericanas; lo cual les exige desarrollar o utilizar mecanismos formales efectivos de monitoreo sistemático del entorno tecnológico y competitivo. Ésto es, la obtención, organización, análisis y poner la información en acción. Este proceso de agregación de valor estratégico facilita al equipo gerencial la identificación de información útil y desplegar estrategias competitivas para mantenerse en el mercado.

Palabras claves: Inteligencia competitiva, estrategia, decisión, acción empresarial, tecnología de información, ética.

\section{Competitive intelligence, strategy and competitiveness in the venezuelan industries}

\section{Abstract}

This article describes what the author views as the context of the new organizational realities; the meaning and the importance of Competitive Intelligence (Cl); its relationship

Recibido: 27-02-97. Aceptado: 04-06-97

Lic. en Felaciones Industriales (UCAB). Especialista en desarrollo organizacional (UCAB). Candidato al Ms. Sc. en Gerencia Empresarial (UCV). Programa de Gerencia Estratégica de RRHH (Electricite de France). Programa Avanzado de Consultoría (ILOS). Consultor de Organización y Estrategia. Miembro de la Society of Competitive Intelligence Professionals (SCIP).

P.O. Box 2299. Caracas - Venezuela. 
with strategic management; The description of the $\mathrm{Cl}$ model process; $\mathrm{Cl}$ implantation guidelines; the importance of the ethical aspects of the process, and the conclusions. This approach is based on the new tecnological, economical and management realities of the venezuelan and latinamerican companies, which urges them to implement or use formal and effective mechanisms of monitoring the competitive and tecnological environment systematically. This is the gathering, organization, analysis and transformation of the information into action; this strategic additive value process helps management teams to identify insightful information and deploy competitive strategies to keep up with the market.

Key words: Competitive intelligence, decision, organizational action, information thecnology, ethics.

\section{Introducción}

Los signos de los tiempos nos orientan por el camino de la obtención de mejores formas de conducción de nuestras empresas. Hoy más que nunca, la administración se encuentra en un ambiente de reflexión, de autogeneración de conceptos y tecnologias que permiten clarificar, de alguna manera, la dinámica del entorno y su impacto en las empresas.

La competitividad global, la reestructuración, desaparición y surgimiento de nuevas estructuras, escasez de recursos y rediseño de procesos, hacen que las energías organizacionales se centren en la gerencia del cambio, mayor atención y cumplimiento de los requisitos del cliente, énfasis en la gerencia de costos y en la calidad de los productos y servicios. Estas tendencias forman parte de la complejidad en la que se encuentra Venezuela y por ende, sus empresas en los distintos sectores; ante lo cual estamos en to que el autor Drucker P. (1992) denominó la sociedad de adversarios.

Ante estas áreas de interés, ya no es suficiente estar informados como actividad pasiva, hay que posicionarse en una actitud proactiva ante los cambios del entorno en que se denvuelven las organizaciones, en lo que los autores como Porter M. (1980), Fuid L. (1988), Gordon I. (1991) y Peña $G$ (1992), han denominado, inteligencia comercial o inteligencia tecnoeconómica y más recientemente, Olivera A. (1996) inteligencia estratégica. Sin embargo, para los efectos de este artículo se utilizará el término de Inteligencia competitiva (IC).

Es así que en el presente artículo, primero, se ubicará en el contexto de las nuevas realidades administrativas en nuestro país y la importancia del análisis del entorno; en segundo lugar, se clarificará el significado de inteligencia competitiva como actividad sensora y alimentadora de la acción gerencial; tercero, la IC en el marco de la formulación de la estrategia; cuarto, se presenta una proposición de un modelo del proceso de $1 \mathrm{C}$; quinto, la importancia de la tecnología de información en el proceso de IC; sexto, se establecerán algunas pautas o lineamientos guía para el funcionamiento de un equipo de apoyo o una unidad de IC como soporte en la toma de decisiones para la conducción exitosa de nuestras 
organizaciones; séptimo, se tratará la cuestión ética en la ejecución del proceso y por último, se presentarán las conclusiones del tema.

\section{El contexto de las Nuevas Rea- lidades Administrativas.}

Con el objeto de ubicar y comprender el marco de actuación de las empresas, se hará mención al conocimiento como fuente de riqueza; la apertura de los mercados nacionales y al procesamiento de información del entorno como fuente de energía organizacional.

En primer lugar, el tránsito de una sociedad industrializada hacia una sociedad informatizada ha implicado un cambio en la conducción de nuestras empresas y en especial hacia la gente como activos humanos poseedores del componente básico en la construcción y mantenimiento de ventajas competitivas, como lo es el conocimiento. En este sentido el autor Toffler A. (1990) fundamentó su obra y argumentaba que los conocimientos guardados con tanto celo por los especialistas se les han escapado de las manos y ya están en el gran público; en esta misma línea se encuentran los grandes grupos empresariales. $Y$ a medida que el conocimiento es redistribuido, también lo es el poder basado en él. De allí que el nuevo sistema para crear riqueza se fundamenta en la mente; es decir, en la productividad de los trabajadores del conocimiento y del servicio, cuyo objeto de trabajo es la información. Entre los cuales destacan, los profesionales que realizan labores de inteligencia competitiva, planificadores, ejecutivos, científicos sociales, ingenieros del conocimiento, educadores, comunicadores sociales e ingenieros.

En segundo lugar, Venezuela ya ha entrado en el escenario de la competitividad, para algunos de una manera evolutiva y para otros, de una manera más progresiva. El proceso de apertura comercial ha implicado cambios en los ámbitos político, económico, laboral y social; nótese, por ejemplo, los cambios en el sector bancario, en el que se encuentra presente más de un $40 \%$ de la banca internacional; el sector petrolero es otro ejemplo de la complejidad y el impacto en las empresas del área e inclusive en cuanto a los requerimientos de recursos humanos especializados y en la infraestructura de servicios; el sector eléctrico se encuentra en la fase de preparación del marco jurídico-legal para la separación de los servicios y por ende, la incorporación de la competencia internacional.

Todo ello está halando a las empresas del sector privado y al sector público a rediseñar y hasta cambiar sus procesos de gestión y de producción.

La visión de procesos está emergiendo sobre el enfoque funcional y divisional; se denota un mayor énfasis en poseer una alta capacidad de respuesta y flexibilidad ante los requerimientos $y$ requisitos de los clientes. Asimismo, las consecuencias para la administración de los recursos humanos son importantes; por ejemplo, la reforma de la Ley Orgánica del Trabajo y la tendencia a una mejor preparación de los trabajadores(en especial, los mencionados anteriormente) en base a sus competencias. El funcionamiento por procesos está exigiendo ma- 
yor énfasis en el trabajo en equipo y a ejecutar múltiples roles en la actuación laboral. Esto último, implica que las empresas preparen y desarrollen a su gente para esta nueva forma de trabajo; aún más, hoy más que nunca, la alianza sector empresarial - sector educativo, es una necesidad imperante como plataforma de trabajo para contar con un equipo humano preparado para interactuar en un escenario competitivo y una permanente actualización e inovación industria-educación.

Adicionalmente, muchas empresas se encuentran evaluando e incorporando herramientas para ordenar, clarificar y gerenciar de mejor manera sus costos, sus procesos y sus mismas estructuras (organizaciones planas e interconectadas). Terminologías como el redimensionamiento organizacional, contratación externa de procesos de apoyo, colocación externa de personal y rediseño de procesos de negocio están en pleno proceso de aprendizaje, mediante la evaluación y adaptación en su ejecución, de acuerdo a nuestra realidad cultural.

En tercer lugar, nos encontramos en una sociedad basada en la información y en el conocimiento como fuente de poder y de riqueza, tal como fue mencionado por Toffler A. (1990); siendo así que, el entorno en el que se desenvuelven las empresas, genera una inmensa gama de datos, hechos o eventos, que al darle significado en función de la misión, objetivos y estrategias de la empresa, se convierten en información valiosa, que servirá de marco de referencia para la identificación de consecuencias para las operaciones presentes y futuras de la organización, creando una base o repertorio de decisiones. Decisiones que al ejecutarla o imprimirle una conducta de acción, se producen resultados que influyen en la dinámica del entorno, ya sea obteniendo mayor posicionamiento o perdurabilidad de la empresa en el tiempo (ver Grático No. 1).

Ante este contexto, se presenta un conjunto de categorías para describir las características de la organización actual $y$ las de la nueva empresa en la cual estaremos conduciendo nuestros propios destinos y los del país como nación dentro del contexto mundial. A continuación se muestra una categorización fundamentada en el autor Tapscott D., Caston A. (1995):

\section{CATEGORIAS}

Estructura

Alcance

Recurso Ppal.

Estado

Punto Ctral.

Motivadores

Dirección

Bases de Acción

Motivación Person
ORG. ACTUAL

Jerárquica

Interna/cerrada

Capital

Estable

Jefes

Premio/castigo

Control admitivo

Control

Satisfacer Superiores
NUEVA EMPRESA

Plana

externa/abierta

Gente/conocimientos

Dinámico/cambiante

Profesionales

Compromiso

Autoadministración

Empoderamiento

Logros en equipos 
Aprendizaje

Compensación

Relación

Actitud

Exigencias
Trabajos especif

Posic. Jerárquica

Competitiva

Indiferencia

Admon. dominante
Múltiples capacid.

Nivel de competencia

Redes

Identificación

Coaching/iderazgo
La importancia de las iniciativas de instrumentar y mantener un proceso de IC dirigidas al logro del éxito empresarial e institucional se fundamentan en su contribución a:

a.- Desarrollar, mejorar e innovar los procesos productivos y los servicios de manera tal de consolidar la capacidad de la organización de perdurar en el tiempo; es decir, contribuir con la viabilidad de la empresa. b.- Mantener la estabilidad de la empresa en un estado de situación de alta turbulencia ambiental, mediante la reducción de incertidumbre en una actualización progresiva y contínua de sus estructuras (estrategias, objetivos, políticas, normas y esquemas organizativos entre otros).

c. Competir exitosamente por la obtención y disponibildad de recursos: capital, tiempo, equipos y materiales, gente, información y conocimientos. Ventocilla E., Alfonso R. (1995).

\section{Gráfico No. 1}

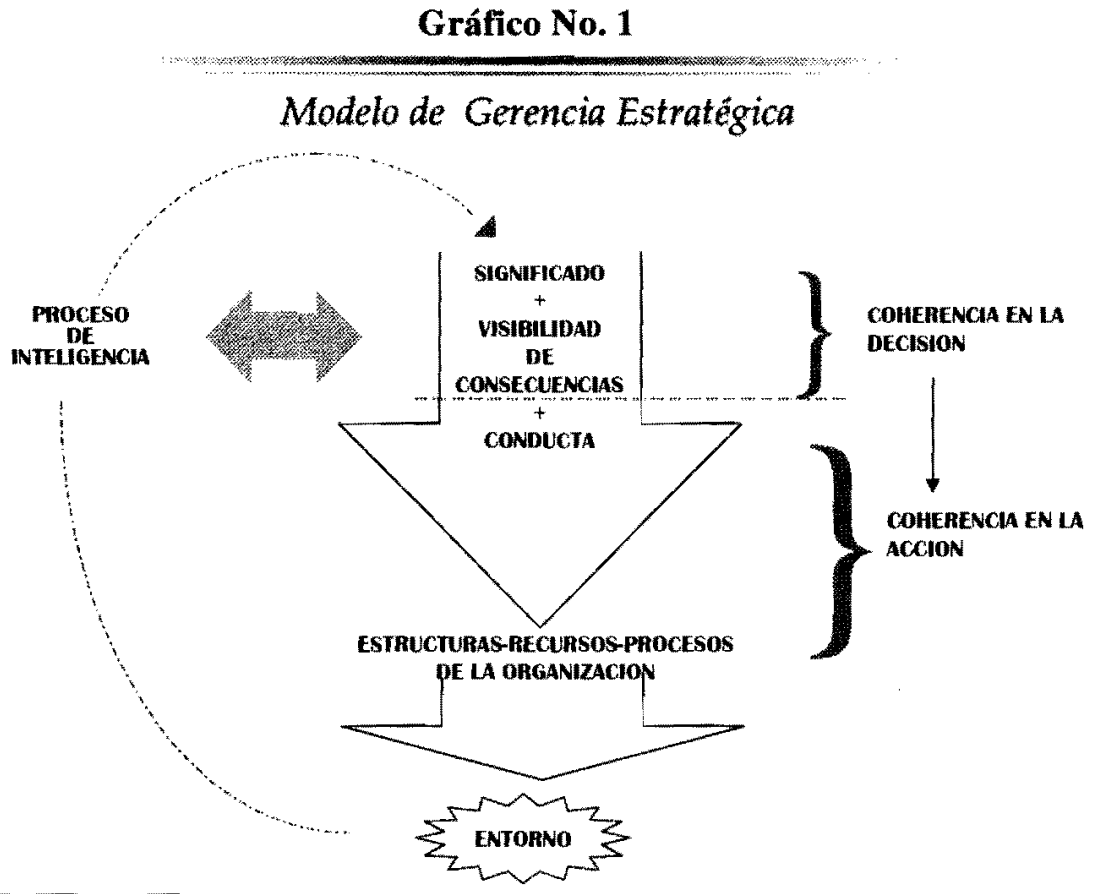


Ahora bien, las empresas deberán desarrollar una serie de capacidades que brindan el soporte de los aspectos mencionados y de acuerdo a Cubillo (1991), estas comprenden:

- Una capacidad de percepción de los factores del ambiente externo; es decir, el desarrollo de mecanismos que permitan detectar y evaluar, con suficiente anticipación, las oportunidades y amenazas para la empresa. Esto incluye por ejemplo, la capacidad para dar respuesta a interrogantes tales como: Qué están haciendo los competidores y qué son capaces de hacer?; Cuáles son las premisas o suposiciones bajo las cuales ellos operan?; Cuáles son las probabilidades de que ocurran nuevos desarrollos en el campo tecnológico?, para qué fecha? y cuál será su impacto en el sector?

- Una capacidad de percepción de los factores del ambiente interno, tales como el clima organizacional, la situación financiera y la capacidad de endeudamiento de la empresa, las habilidades y destrezas de los activos humanos y sus necesidades de formación y entrenamiento y otros, los cuales determinan en gran medida, las debilidades y fortalezas de la empresa o institución.

- Una capacidad para anticipar la probabilidad de escenarios, la evolución de problemas y de las soluciones asociadas.

\section{Significado de Inteligencia Competitiva.}

El conocimiento de la realidad externa de la empresa, asi como el procesamiento y evaluación de la información que alimenta la toma de decisiones para la acción organizacional se lleva a cabo mediante una actividad interna de seguimiento, la cual permite obtener una base de conocimientos sobre los cambios que se suceden en el entorno. Ya los autores Katz D., Khan R. (1977) señalaban su importancia al concebirla como inteligencia o función retroalimentadora. Adicionalmente, sostuvieron que la consideración misma de la organización como un sistema cerrado, destacaba lo débil que resultaban muchas compañías industriales en sus departamentos de investigación de mercados, siendo que dependen, al extremo, de este último. A su vez, consideraron la tendencia de que en nuestra sociedad las organizaciones se irán moviendo cada vez más hacia el mejoramiento de las condiciones de investigación para valorar las fuerzas del mercado.

Es por ello que la consideración de la relación de la empresa con los clientes, el conocimiento de los movimientos de las empresas competidoras, sirve de referencia para la investigación del mercado (empresa-clientes); la investigación del mercado competitivo (clientes-competidores) y el desarrollo de la inteligencia competitiva (empresa-competidores). (Ver gráfica 2).

En línea con lo anterior, Porter $M$. (1980) propone que la formulación de una estrategia competitiva implicaba posicionar a la empresa para maximizar el valor de las capacidades que la distinguen de sus competidores. Un aspecto central en ello es, el análisis perceptivo de la competencia, cuyo objetivo es el de desarrollar un perfil de la naturaleza y éxito de los cambios de estrategia que cada competi- 
dor podría hacer, la respuesta probable de cada competidor a la gama de posibles movimientos estratégicos que otras empresas pudieran iniciar, y la reacción probable de cada competidor al arreglo de cambio en el sector industrial y los cambios del entorno que pudiera ocurrir.

La noción de inteligencia competitiva ha recibido diversas definiciones, entre las que se pueden senalar la de Lagerstam C. (1989), quien la consideró como todas aquellas cosas que deben conocerse de antemano, previo al inicio de un curso de acción. Cubillo J. (1991) la define como "aquellas capacidades intelectuales organizadas de una empresa productora $\circ$ de un departamento gubernamental para percibir cambios emergentes en las condiciones de su ambiente operativo y para preparar movimientos alternativos adaptativos, a fin de maximizar la probabilidad de obtención de objetivos, en virtud de esos cambios.

Considerando el aporte de autores como Olivera A. (1996), Ventocilla E. (1995), Peña G. (1992) y otros, asi como la experiencia del autor del presente papel de trabajo, la inteligencia competitiva podría definirse como el proceso sistemático de identificación, procesamiento, evaluación y determinación de impactos y consecuencias, basado en datos públicamente disponibles, de la evolución interna y externa de un sector empresarial dado, las capacidades y el comportamiento de los competidores, proveedores

\section{Gráfico No. 2}

\section{Modelo de Inteligencia Competitiva}

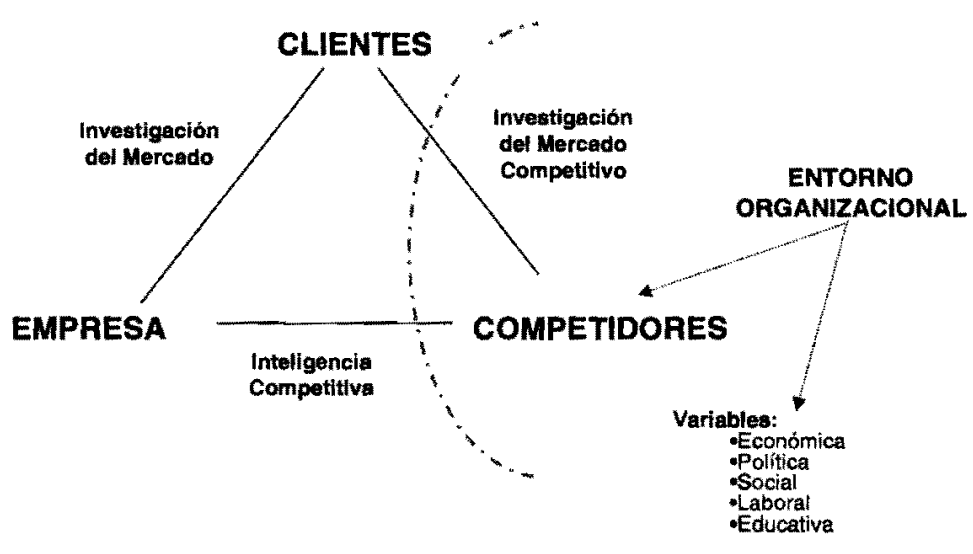

Fuente Adaptedo de Ian Gardon (1toen) 
y clientes actuales y potenciales, a fin de construir una base de decisiones que apoye las acciones de viabilidad, estabilidad y factibilidad de la organización en el tiempo.

Si detallamos la definición presentada, tenemos que es:

a. Proceso Sistemático: debido a que obedece a un conjunto de fases o etapas interrelacionadas y secuenciales que de manera contínua y de acuerdo a una metodología formal o producto de la experiencia y el aprendizaje organizacional, facilita la captura y visualización de las consecuencias de los datos e información provenientes de los eventos que se suceden en el entorno y que estén públicamente disponibles.

b. Identificación, procesamiento, evaluación y determinación de impactos: el cual hace referencia a la determinación cuantitativa y cualitativa de la ocurrencia de eventos que pudieran influir en las decisiones y acciones de la empresa en el futuro, así como sus interrelaciones bajo un enfoque de análisis y síntesis, a fin de construir escenarios y evaluar alternativas de decisión, previa a la acción organizacional.

c. Evolución interna y externa de un sector empresarial: la cual está relacionada con la comparación de información cuantitativa y cualitativa durante el desarrollo o desenvolvimiento de una organización en el tiempo.

d. Capacidades y comportamiento de los competidores: hace referencia a la obtención y actualización de información asociada a las habilidades, recursos y estrategias como un patrón de comportamiento observable (Villalba J. 1996), por parte las empresas competidoras actuales y potenciales. De igual forma, es conveniente conocer las capacidades y el comportamiento de los clientes y de los proveedores, actuales y potenciales.

e. Base de decisiones: se refiere al conjunto o repertorio de alternativas disponibles posibles de convertirse en cursos de acción al ser autorizados por el equipo gerencial de la organización.

\section{Estrategia e Inteligencia Com- petitiva.}

Cualquier organización, como todos los sistemas sociales, necesitan desarrollar y desplegar el grado de su aceptación en el entorno y de respuesta a los cambios que se producen en él, a fin de asegurar la disponibilidad de los recursos pertinentes para su sobrevivencia. Lo cual conlleva a alcanzar objetivos de:

a. Desarrollo y adaptación interna,

b. Influencia y competencia externa, $y$ de

c. Búsqueda de segmentos protegidos en el mercado,

con la finalidad de modificar o ajustarse a las situaciones del entorno, éstas constituyen la razón de la estrategia. Ventocilla E., Alfonso R. (1995).

El autor Bamard C. (1957) sostuvo que la causa de la inestabilidad y la duración limitada de las organizaciones formales es causada por fuerzas externas. Por ello, en este papel de trabajo se entenderá el término estrategia, como el conjunto de decisiones y acciones llevadas a cabo por las empresas para modificar en forma positiva su estado de situación, asegurando el mantenimiento de sus reglas constitutivas y para crear con- 
diciones favorables para el logro de su misión y objetivos. Se busca entonces, lograr un elevado nivel de coherencia entre las decisiones y las acciones en un entorno de cambios evolutivos a velocidades revolucionarias. (Ver gráfica 1).

La IC se constituye como soporte fundamental en la formulación de las estrategias al brindar una base de conocimientos y de decisiones que le permite decidir y actuar en forma oportuna. Lo anterior es reforzado según un reciente estudio llevado a cabo por una empresa consultora venezolana, el cual arrojó como resultado que, en una muestra de 40 empresas de las 500 empresas más grandes del sector privado, el $90 \%$ realiza el análisis de los competidores obedeciendo a un proceso de planificación estratégica. Perdomo L. (1996).

La IC puede ubicarse en el marco del proceso de formulación de estrategias en la fase de lo que los autores han denominado análisis ambiental, auditoria externa e interna, analisis del contexto; considerando que la inteligencia va más allá de la identificación de las oportunidades, amenazas, fortalezas y debilidades, - la identificación de tendencias.

\section{Modelo del proceso de Inteli- gencia Competitiva.}

Basado en los aportes de Peña G. (1992), la generación de inteligencia al ser considerada como un proceso contempla las siguientes fases, las cuales se explicarán brevemente. (Ver gráfica 3 ).

a.- Identificación de los Requerimientos de Información y Priorización de Atención: esta fase se fundamenta en la elaboración de un registro de la inteligencia requerida por las unidades internas de la organización, de manera tal de obtener un perfil de áreas de interés. Por otro lado, es importante conocer, el tipo de información disponible y sus fuentes; quiénes pueden actuar como puntos de enlace de información o expertos en determinados campos del conocimiento; así como un diagnóstico de los canales de comunicación de la empresa para su utilización en la diseminación selectiva de información. Una vez completado el proceso de identificación de requerimientos de información, se procede a la determinación de prioridades en cuanto a cuáles requerimientos se deben atender primero, de acuerdo al cumplimiento de una serie de criterios preestablecidos.

b.- Determinación de Objetivos: cada requerimiento de información detectado se convierte en un proyecto de inteligencia, el cual apuntará a determinados objetivos, ya sean éstos de reconocimiento (conocer mejor el sector o las acciones que adelantan los competidores), ofensivos (evaluación de fortalezas, debilidades y las probables respuestas de los competidores) y defensivos (anticipar los movimientos de los competidores).

c.- Recolección de Datos: está alineada en función de los objetivos establecidos; para lo cual es pertinente la identificación de las entidades, las variables, las fuentes y la forma como vienen los datos einformación relevante (lo que permite definir la metodología y la tecnología de recolección a utilizar). Debe destacarse que la inteligencia competitiva trabaja sobre datos $e$ información públicamente disponibles. 
Gráfico No. 3

\section{Modelo de la Inteligencia como Proceso}

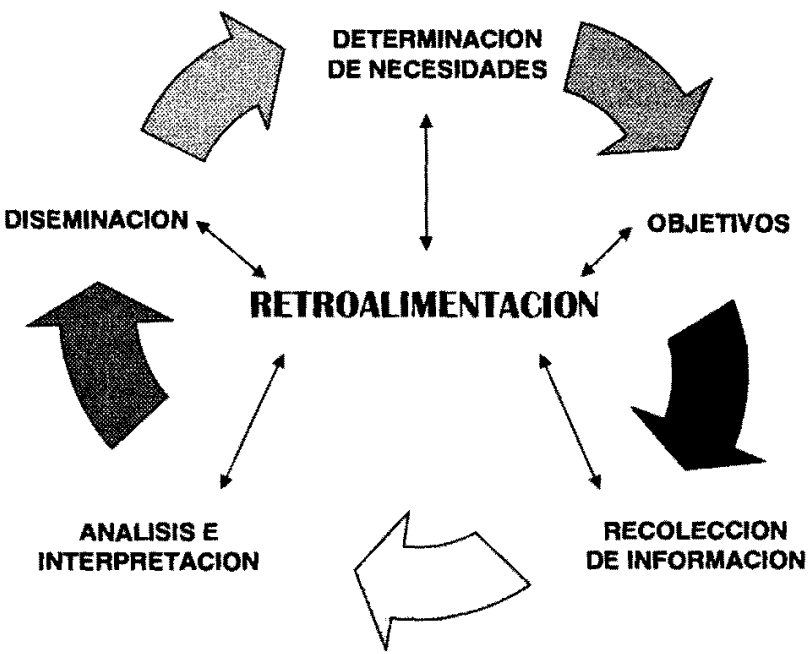

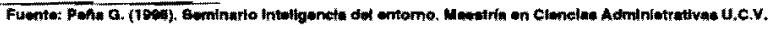

d.- Procesamiento de Información: hace referencia al otorgamiento de significado a los datos para convertirlos en información que, al visualizarse identifica sus consecuencias para la organización, se transforma en conocimiento. Es asi que, para comprender la información o el desarrollo de los eventos, ella debe ser analizada en su contexto y relacionada con los acontecimientos previos o con eventos que influencien la interpretación. De esta manera se estaría construyendo una base de decisiones cuya ejecución, mediante una conducta, pasaría a convertirse en una acción organizacional con unos resultados que impactan en el entorno. Ventocilla E. (1996). La utilización de técnicas cuantitativas y cualitativas es clave en esta fase del proceso. e.- Diseminación Selectiva de Información: para Peña G. (1992), un sistema de distribución de inteligencia considera el uso de informes, de carteleras, salas de demostración, boletines y reuniones. La frecuencia y contenido y el formato de los informes, variarán según la inteligencia a diseminar; entre éstos se encuentran: Informes de inteligencia crítjca.- especificos en contenido, breves y descriptivos y que requieren de acción inmediata; de situación.- de cobertura limitada y su propósito es informar sobre actividades de los competidores y sobre cambios en el ambiente (interno y externo) y los informes solicitados por los usuarios.

f.- Evaluación del Proceso de Inteligencia: hace referencia a la verificación 
y seguimiento de la inteligencia distribuida; por to que el verdadero reto de este proceso es el de desembocar en una acción apoyada o guiada por los productos y servicios de la inteligencia competitiva. Por otro lado, implica un proceso de contínua retroalimentación a fin de ir mejorando en forma sistemática sus resultados.

\section{Tecnología de Información e Inteligencia Competitiva.}

Aunque los sistemas de almacenamiento de inteligencia no necesariamente tienen que estar automatizados, la disponibilidad de las bondades de una tecnología de información más rápida (clienteservidor) y más económica, se hacen cada vez más necesaria; así como bases de datos que integren textos, sonido e imagen para manejar datos e información que de otra forma sería imposible.

Tal como afirman Tapscott D., Caston A. (1995), el nuevo paradigma de la tecnología de información va paralelo a los múltiples cambios y tendencias, ya que es abierta, e interconectada; modular y dinámica. Con base en partes intercambiables y que tecnológicamente posibilita la distribución de inteligencia y la toma de decisiones para los clientes internos de la organización.

En la actualidad existen diversos programas de inteligencia de negocios (Business Intelligence softwares), los cuales brindan opciones de procesamiento analítico en linea y sistemas de soporte para la toma de decisiones (Decision Support Systems). Entre las aplicaciones de avanzada se encuentra la construcción de redes de capacidades neurales.

Por otro lado, existen en nuestro país, empresas de consultoría afiliadas al Instituto Latinoamericano de Organización y Sistemas (ILOS) que están desarrollando aplicaciones asociadas a la Ingeniería del Conocimiento, utilizando softwares para el adiestramiento basado en computadores y el desarrollo de sistemas electrónicos de soporte al desempeno. La idea es mantener una actualización permanente en el estado del arte de la tecnología, tanto en los campos de la epistemología, la psicología cognitiva y los sistemas de aprendizaje basado en computadoras, como el desarrollo de las habilidades requeridas para la aplicación de las nuevas herramientas basadas en la información y el conocimiento.

\section{Lineamientos para la Instru- mentación del Proceso de IC.}

Las siguientes son algunas pautas - guias orientadoras asociadas a la instauración de un proceso de $\mathrm{IC}$ en las organizaciones:

a. Todo empleado de la organización es parte del proceso de IC de la empresa; la vigilancia de los competidores es un esfuerzo del negocio.

b. El equipo o la unidad de IC reportará al más alto nivel ejecutivo y deberá estar abierta a recibir información por múltiples vías sobre cualquier paso $o$ acción que ejecuten o se sospeche que efectúe aquellas empresas consideradas como de la competencia. 
c. El equipo gerencial de la organización (gerentes y supervisores) forma parte de la unidad de IC.

d. El equipo o unidad de lC contará con un sistema de información basado en la ingeniería del conocimiento y en los sistemas de soporte para la toma de decisiones, los cuales brindarán apoyo a las acciones estratégicas de la organización.

e. El equipo o unidad de IC establecerá los criterios para la elaboración de los programas de custodia, conservación y protección de la información vital de las operaciones y del mercado de la empresa; fortaleciendo $e$ instalando la cultura de la seguridad de la información de la organización.

f. Los productos que se generen como resultado de los planes y proyectos de la $1 \mathrm{C}$ tendrán rango de información confidencial, quedando prohibida su divulgación fuera del recinto de la empresa.

g. Las actividades del equipo o la unidad de $\mathrm{IC}$ se fundamentará en principios éticos en la ejecución de sus planes y programas de acción.

\section{La Etica en el Proceso de Inte- ligencia Competitiva.}

Si bien es cierto que la preparación de estrategias superiores exige información competitiva de calidad, se podría sentir la tentación de obtenerla con base en cualquier fuente disponible. Por lo que es necesario establecer una línea divisoria entre inteligencia competitiva y espionaje industrial o comercial; es por ello, que la definición indicada en este trabajo hace énfasis en la recopilación, procesamiento y diseminación de datos e infor- mación públicamente disponibles. En este sentido, Gordon I. (1991) sostiene que la disponibilidad pública de los datos es lo que distingue los dos aspectos; además, si cualquiera puede legaimente recopilar los datos, sabiendo solamente dónde mirar, qué preguntar y cómo preguntar sin mal interpretarse, la actividad entonces se considera como legitima.

Por su parte, Fuld L. (1990) argumenta que, el sentido común, la creatividad y la persistencia, son los ingredientes para un proyecto o programa de inteligencia competitiva exitoso; sin embargo, aquellos que participan en la recolección de datos e información afrontan situaciones tentadoras para actuar ilegal o inmoralmente, razón por la cual se debe conocer la normativa legal como por ejemplo, la Ley Orgánica del Trabajo que en su Título II de la Relación de Trabajo, Capítulo VI, artículo 102 , literal $h$, considera como una de las causas justificadas de despido la revelación de secretos de manufacturas, fabricación o procedimiento. En igual forma, todo lo referente a la Ley de Propiedad Industrial y Derechos de Autor.

En todo caso, las situaciones que ponen en duda y en peligro la fortaleza moral son infinitas, y como cada uno de nosotros poseemos valores distintos, es harto dificil preescribir reglas de conducta; por lo que es importante hacerse las siguientes preguntas cada vez que nos encontremos ante una situación de este tipo:

- Necesito realmente esta información para tomar una decisión sana?

- Cuánto me ayudará a mi o a mi organización esta nueva información? 
- He agotado todos los medios claramente éticos?

- Para mis adentros, qué creo que es bueno o malo?

Finalmente, debe destacarse que una conducta éticamente y moralmente deficiente puede ser demoledora para la imagen de una organización; asi como el acopio de información sin ética puede afectar la salud, el capital, apoyo político y el bienestar de la organización.

\section{Conclusiones}

1. Un programa de inteligencia competitiva empresarial está lejos de ser algo muy complejo y complicado que sólo un grupo reducido de empresas puede darse el lujo de mantener; sin embargo, todas las empresas y en especial, las pequeñas y medianas empresas, necesitan instrumentar urgentemente un programa de inteligencia competitiva aunque sea sencillo, que permita hacer un seguimiento de un entorno caracterizado por la competitividad, apertura a la inversión internacional, con la consecuente eliminación del proteccionismo estatal.

2. Se debe hacer un mayor énfasis de las aplicaciones más utilizadas en los programas de inteligencia competitiva, como son los de la caracterización del perfil de situación y de comportamiento del sector industrial en el cual se desenvuelva la empresa, la técnica de comparación con el mejor de la clase o Benchmarking y la elaboración de perfiles de personalidad de los proveedores y de los competidores.

3. Los proyectos y programas de inteligencia competitiva deben estar in- mersos en el proceso de formulación de las estrategias de las empresas y en el cual participen y se involucren gerentes y supervisores de las áreas clave de la organización con el apoyo de un equipo integrado por la gente de mercadeo, ventas, planificación estratégica, finanzas, recursos humanos y comunicaciones corporativas.

4. La incorporación de las herramientas proveniente de los avances de la tecnología de información es clave para obtener el mejor provecho del almacenamiento, diseminación y una oportuna y conflable capacidad de respuesta de la inteligencia competitiva.

5. La instauración de un proceso de inteligencia competitiva debe fundamentarse en lo posible, en un modelo que de claridad a las acciones de sus proyectos y programas, en el cual se indique su estructura, recursos y procesos.

6. La ejecución de los proyectos y programas de inteligencia competitiva deben regirse por la normativa legal, siguiendo los principios éticos en la recolección de datos e información.

\section{Referencias Bibliográficas.}

Barnard, Chester. (1956). The Functions of the Executives.USA.Harvard University Press. p.6.

Cubillo, Julio. (1991). Thecno-economic intelligence. Viena. Unido.

Drucker, Peter. (1990). Las Nuevas Realidades Administrativas.Colombia. Edit. Norma. p.2-18.

Fuld, Leonard. (1990). Inteligencia Comercial: Técnicas para Analizar la Competencia. Colombia. Edit. Legis. p.171-179. 
Gordon, lan. (1989). Cómo Anticiparse a la Competencia. Colombia. Edit. Legis. p. 1-26.

Katz Daniel, Khan Robert. (1977). Psicologia Social de las Organizaciones. México. Edit. Trillas. p. 37.

Lagerstam, Catherina. (1981). The Theory of Business Intelligence: The Intelligence Process. Yugoslavia. Ponencia del Seminario "Information Industries Knowledge".

Olivera, Angel. (1996). Modelo de Análisis del Entorno. Mimeografiado.

Peña, Gustavo. (1992). "La Inteligencia Tecnoeconómica: Una Mirada al Futuro". En: Revista Espacios. ConicitVenezuela. Vol. 13. No. 2. p. 21-28.

Perdomo, Ligia. "Industrias Acostumbran a Medir a sus Competidores". En: Economia Hoy. Año 8. No. 2.236. 12 de Junio. 1996. p 11.

Porter, Michael. (1980). Estrategia Competitiva: Técnicas para el Análisis de los Sectores Industriales y de la Competencia. México. Edit. Cecsa. p. 9-53.
Romero García, Oswaldo. (1994)." Outplacement: Una Experiencia Venezolana". En: Memorias EVEMO 5. p.075-084.

Tapscott Don, Caston Art. (1995). Cambio de Paradigmas Empresariales. México. Edit. MacGraw Hill. p.XI-30.

Toffler, Alvin. (1990). El Cambio del Poder. Powershift: Conocimientos, Bienestar y Violencia en el Umbral del Síglo XXI. Madrid. Edit. Plaza \& Janes Editores. p.31.32.

Ventocilla Eliodoro, Alfonso Roque. (1995). Programa Avanzado de Consultoría. PAC. Venezuela. Ediciones del Instituto Latinoamericano de Organización y Sistemas (ILOS). p. III-17,18.

Ventocilla, Eliodoro. (1995). "Tiempo, Lenguaje y Cambio". Papel de trabajo. Edición Mimeografiada. ILOS. p.14.

Villalba, Julián. (1996). Menú Estratégico: EI Arte de la Guerra Competitiva. Venezuela. Edic. IESA-Centro Nacional para la Competitividad. p.13-105. 